\title{
Electrical properties and conduction mechanisms in La2/3Ca1/3MnO3 thin films prepared by pulsed laser deposition on different substrates
}

\begin{abstract}
Perovskite manganite $\mathrm{La} 2 / 3 \mathrm{Ca} 1 / 3 \mathrm{MnO} 3$ thin films were directly grown on $\mathrm{MgO}(100)$, $\mathrm{Si}(100)$ and glass substrates by pulsed laser deposition. From the XRD patterns, the films are found to be polycrystalline, single-phase orthorhombic. The metal-insulator transition temperature is $209 \mathrm{~K}$ for $\mathrm{LCMO} / \mathrm{MgO}, 266 \mathrm{~K}$ for $\mathrm{LCMO} / \mathrm{Si}$ and $231 \mathrm{~K}$ for film deposited on the glass substrate. The conduction mechanism in these films is investigated in different temperature regimes. Low-temperature resistivity data below the phase transition temperature ( $\mathrm{T}$ P) have been fitted with the relation $f=f 0+f 2 \mathrm{~T} 2+f 4.5 \mathrm{~T} 4.5$, indicating that the electron-electron scattering affects the conduction of these materials. The high-temperature resistivity data $(\mathrm{T}>\mathrm{T} \mathrm{P}$ ) were explained using variable-range hopping (VRH) and smallpolaron hopping (SPH) models. Debye temperature values are $548 \mathrm{~K}$ for $\mathrm{LCMO} / \mathrm{Cg}, 568 \mathrm{~K}$ for $\mathrm{LCMO} / \mathrm{Si}$ and $508 \mathrm{~K}$ for $\mathrm{LCMO} / \mathrm{MgO}$ thin films. In all thin films, the best fitting in the range of VRH is found for 3D dimension. The density of states near the Fermi level N (E F) for $\mathrm{LCMO} / \mathrm{MgO}$ is lower due to the prominent role of the grain boundary in $\mathrm{LCMO} / \mathrm{MgO}$ and increase in bending of $\mathrm{Mn}-\mathrm{O}-\mathrm{Mn}$ bond angle, which decreases the double exchange coupling of $\mathrm{Mn} 3+-\mathrm{O} 2-\mathrm{Mn} 4+$ and in turn makes the LCMO/MgO sample less conducting as compared to the other films.
\end{abstract}

Keyword: La2/3Ca1/3MnO3 thin films; Pulsed laser deposition; Thin films; Perovskite manganites 